\title{
LETTERS
}

\section{Anesthesia assistants - a failed solution since 2005}

In a letter response regarding their Analysis article, ${ }^{1,2}$ Drs. Orser and Wilson state that "In Canada, certified anesthesia assistants support and work under the supervision of anesthesiologists. The Government of British Columbia recently expanded its training program for certified anesthesia assistants to address the shortage of anesthesia providers in that province."

This would imply that anesthesia assistants are trained, certified and regulated to independently provide anesthesia care throughout the perioperative experience, but anesthesia assistants in Canada are not anesthesia providers. As far as I am aware, anesthesia assistants (particularly in Ontario), assist the anesthesiologist in the sense that they complete the anesthesia machine checks in the morning, set up table tops, assist with the regional administration of anesthesia and are a second set of hands in anticipated difficult airways. They are not anesthesia providers, and this is not a solution or a strategy for the disparity in anesthesia services or the shortage of anesthesiologists.

Drs. Orser and Wilson also mentioned that "Canada has already invested in the high-quality educational infrastructure needed to train family physician anesthestists, and the demand from keen physicians for provincially funded training positions far exceeds our current training capacity." This is an interesting comment, considering there is also a family physician shortage in many provinces, especially in rural areas - a shortage that has been partially alleviated thanks to nurse practitioners in other jurisdictions. Family physicians commonly have a heavy workload in rural areas, including emergency department coverage, as well as attending to the needs of their large patient rosters. Growing up in rural Ontario, I had a family physician who had office hours to attend to his patient roster, covered the emergency department, was the coroner on call for the area and provided the occasional general anesthetic. I cannot see how adding anesthesia to the repertoire of family physicians is a viable solution.

What is a viable, cost-effective, efficient and safe solution to the anesthesiologist shortage and service disparities in rural communities? The answer is the certified registered nurse anesthetist (CRNA). ${ }^{3}$ CRNAs are advanced practice nurses who practise with a high degree of autonomy and respect in the United States. Nurse anesthetists have been providing anesthesia care in the US for more than 150 years and safely administer more than 49 million anesthetics to patients each year. CRNAs are the primary providers of anesthesia care in rural areas and offer obstetrical, surgical, pain management and trauma stabilization services; cost efficiency of CRNAs helps control escalating health care costs. Hundreds of Canadian CRNAs currently practising in the US may wish to come home and provide their exceptional anesthesia services to fellow Canadians.
According to a recent Gallup Poll, and for the 18th year in a row, Americans have rated the honesty and ethics of nurses highest among a list of professions. ${ }^{4}$ It is time for the provincial governments to start talking to registered nurses, nurse practitioners and certified registered nurse anesthetists to come up with realistic and viable strategies. For $10-15$ years, it has been frustrating to watch the provinces invest a considerable amount of money into physician-backed ideas that have not been working, and I would argue that the public is starting to notice. It's time for a change.

\section{Josh Booth MSN}

Certified registered nurse anesthetist (CRNA), Ann Arbor, Mich.

Cite as: CMAJ 2020 October 13;192: E1216. doi: 10.1503/cmaj.76785

\section{References}

1. Orser BA, Wilson CR. Canada needs a national strategy for anesthesia services in rural and remote regions. CMAJ 2020;192:E861-3.

2. Orser BA, Wilson RC. The authors respond to "Nurse anesthesiologists" [letter]. CMAJ 2020;192: E1215.

3. Certified registered nurse anesthetists fact sheet. Park Ridge (IL): American Association of Nurse Anesthetists; updated 2019 Aug. 8. Available: www.aana. com/membership/become-a-crna/crna-fact-sheet (accessed 2020 Sept. 16).

4. Reinhart RJ. Nurses continue to rate highest in honesty, ethics. Washington (DC): Gallup; 2020. Available: https://news.gallup.com/poll/274673/ nurses-continue-rate-highest-honesty-ethics.aspx (accessed 2020 Sept. 16).

Competing interests: None declared. 